\title{
Oxygen-enhanced MRI accurately identifies, quantifies, and maps tumor hypoxia in preclinical cancer models
}

James PB O'Connor ${ }^{1,2,3}$, Jessica KR Boult ${ }^{4}$, Yann Jamin ${ }^{4}$, Muhammad Babur ${ }^{5}$, Katherine G Finegan ${ }^{5}$, Kaye J Williams ${ }^{1,5}$, Ross A Little ${ }^{2}$, Alan Jackson ${ }^{2}$, Geoff JM Parker $^{2}$, Andrew R Reynolds ${ }^{6}$, John C Waterton ${ }^{2}$, Simon P Robinson ${ }^{4}$

${ }^{1}$ Institute of Cancer Sciences, University of Manchester, Manchester, UK;

${ }^{2}$ Centre for Imaging Sciences, University of Manchester, Manchester, UK;

${ }^{3}$ Department of Radiology, Christie NHS Foundation Trust, Manchester, UK;

${ }^{4}$ Division of Radiotherapy and Imaging, The Institute of Cancer Research, London, UK;

${ }^{5}$ Manchester Pharmacy School, University of Manchester, Manchester, UK;

${ }^{6}$ Tumour Biology Team, Breakthrough Breast Cancer Research Centre, The Institute of Cancer Research, London, UK

Running title: Imaging tumor hypoxia in vivo with oxygen-enhanced MRI

Key words: Biomarker; Hypoxia; Imaging; MRI; Validation

\section{Grant support:}

1. Cancer Research UK (CRUK) Clinician Scientist award (grant C19221/A15267) to J. P. B. O'Connor;

2. CRUK and EPSRC Cancer Imaging Centre in Cambridge and Manchester funding to The University of Manchester (grant C8742/A18097) to J. P. B. O’Connor, K. J. Williams, A. Jackson, G. J. M. Parker; 
3. CRUK Cancer Imaging Centre funding to The Institute of Cancer Research (grants C1060/A10334 and C1060/A16464) to S. P. Robinson;

4. The Wellcome Trust funding (grant $091763 Z / 10 / Z$ ) to S. P. Robinson;

5. Paul O'Gorman Postdoctoral Fellowship funded by Children with Cancer UK (grant 2014/176) to Y. Jamin.

6. Breakthrough Breast Cancer Senior Fellowship to A. R. Reynolds. Breakthrough Breast Cancer, which has recently merged with Breast Cancer Campaign to form Breast Cancer Now.

\section{Corresponding author details:}

Dr James O'Connor

Institute of Cancer Sciences, University of Manchester

Cancer Research UK Manchester Institute

Wilmslow Road

Withington

Manchester, M20 4BX

UK

Tel: +441614463896

Fax: ++ 1614468532

Email: james.o'connor@manchester.ac.uk

Potential Conflict of Interest: GJMP is a director and shareholder in Bioxydyn Limited. No other authors declared a potential conflict of interest.

\section{Word count 4516}

\section{5 figures and 1 table}




\section{ABSTRACT}

There is a clinical need for non-invasive biomarkers of tumor hypoxia for prognostic and predictive studies, radiotherapy planning and therapy monitoring. Oxygen enhanced MRI (OE-MRI) is an emerging imaging technique for quantifying the spatial distribution and extent of tumor oxygen delivery in vivo. In OE-MRI, the longitudinal relaxation rate of protons $\left(\Delta R_{1}\right)$ changes in proportion to the concentration of molecular oxygen dissolved in plasma or interstitial tissue fluid. Therefore, well-oxygenated tissues show positive $\Delta R_{1}$. We hypothesized that the fraction of tumor tissue refractory to oxygen challenge (lack of positive $\Delta R_{1}$, termed "Oxy- $R$ fraction") would be a robust biomarker of hypoxia in models with varying vascular and hypoxic features. Here we demonstrate that OE-MRI signals are accurate, precise and sensitive to changes in tumor $\mathrm{pO}_{2}$ in highly vascular 786-0 renal cancer xenografts. Furthermore, we show that Oxy-R fraction can quantify the hypoxic fraction in multiple models with differing hypoxic and vascular phenotypes, when used in combination with measurements of tumor perfusion. Finally, Oxy-R fraction can detect dynamic changes in hypoxia induced by the vasomodulator agent hydralazine. In contrast, more conventional biomarkers of hypoxia (derived from blood oxygenation-level dependent MRI and dynamic contrastenhanced MRI) did not relate to tumor hypoxia consistently. Our results show that the Oxy-R fraction accurately quantifies tumor hypoxia non-invasively and is immediately translatable to the clinic. 


\section{INTRODUCTION}

Hypoxia is a common feature of most solid malignancies, resulting from an imbalance between oxygen delivery and consumption (1). Tumor hypoxia is associated with the activation of angiogenesis and with metastatic potential (2). Consequently, tumor hypoxia is an important negative prognostic factor (3-5). Tumor hypoxia also mediates resistance to radiotherapy and to some chemotherapy agents and is an independent predictor of treatment failure. Strategies to counteract tumor hypoxia using either radiosensitizers or hypoxia-activated cytotoxic agents are currently being evaluated (6).

A non-invasive imaging biomarker that identifies the presence of hypoxia and measures its extent and spatial distribution within a tumor would help facilitate personalized medicine. However, no such tool is currently available. Proton $\left({ }^{1} \mathrm{H}\right) \mathrm{MRI}$ is used routinely in clinical medicine, making it an attractive non-invasive modality for measuring oxygen delivery and hypoxia in tumors. Current ${ }^{1} \mathrm{H}$ MRI methods of imaging hypoxia have focused on either dynamic contrast enhanced MRI (DCE-MRI) or $\mathrm{R}_{2}{ }^{*}$ based intrinsic susceptibility imaging, also referred to as blood oxygenation level dependent (BOLD) imaging (7).

In DCE-MRI, administration of a gadolinium-based contrast agent allows estimation of blood vessel flow and permeability, providing an indirect measurement of oxygen delivery and necrosis (8). In BOLD imaging, paramagnetic deoxyhemoglobin molecules in erythrocytes create magnetic susceptibility perturbations around blood vessels, which increase the local transverse MRI relaxation rate $\left(\mathrm{R}_{2}{ }^{*}\right.$; units $\left.\mathrm{ms}^{-1}\right)$. The value of tumor $\mathrm{R}_{2}{ }^{*}$ decreases when blood oxygen saturation increases following inhalation of hyperoxic gas (9). Unfortunately, both DCE-MRI and BOLD imaging have significant limitations which have hindered implementation as clinical biomarkers of hypoxia (10). Neither measure hypoxia directly. Further, BOLD measurements are affected by presence of hemorrhage, by change in vessel geometry and by artifact in air/soft tissue interfaces, such as in the lungs and bowel (7). 
Oxygen enhanced MRI (OE-MRI) is a distinct ${ }^{1} \mathrm{H}$ MRI method for quantifying tumor oxygen delivery. Here, the MRI longitudinal relaxation rate $\left(R_{1}\right.$; units $\left.\mathrm{s}^{-1}\right)$ is sensitive to changes in the level of molecular oxygen $\left(\mathrm{O}_{2}\right)$ dissolved in blood plasma or interstitial tissue fluid $(11,12)$. When hyperoxic gas is inhaled, excess oxygen is carried in the blood plasma in tissues with adequate perfusion. Since well oxygenated tissue has near complete saturation of hemoglobin molecules (13), the excess delivered oxygen remains dissolved in blood plasma and interstitial tissue fluid, where it increases the $R_{1}$ value (Figure 1a). The change in $R_{1}\left(\Delta R_{1}\right)$ observed is theoretically proportional to magnitude of change in dissolved $\mathrm{O}_{2}$ concentration for a given voxel (11) (Figure 1b). Several previous studies have reported positive average values of $\Delta R_{1}$ following oxygen inhalation in preclinical models of cancer (14-22) and in human tumors $(20,23-$ 25). Importantly, while both oxygen relaxivity, baseline $R_{1}$ and signal-to-noise ratios show some variation with field strength, the technique is feasible on both preclinical and clinical MRI platforms $(22,26)$.

Measuring positive $\Delta R_{1}$ in OE-MRI quantifies and maps oxygen delivery by identifying tissue with fully saturated hemoglobin, but does not directly identify tissue hypoxia per se. Tumor sub-regions refractory to oxygen challenge are considered to have low hemoglobin oxygen saturation and so excess delivered $\mathrm{O}_{2}$ molecules bind preferentially to hemoglobin molecules but do not significantly alter plasma $\mathrm{pO}_{2}$ (10) (Figure 1c). Several preclinical OE-MRI studies have reported that some tumor sub-regions are refractory to hyperoxic gas challenge since they had no positive $\Delta R_{1}$ change $(14,19$ 21). If these regions are perfused yet lacking in oxygen enhancement then OE-MRI should identify regions of tumor hypoxia (Figure 1d). In this study, we tested the hypothesis that measuring the fraction of each tumor refractory to oxygen challenge determined by having absent positive $\Delta R_{1}$ (hereafter termed "Oxy- $R$ fraction") - would enable the identification, quantification and mapping of tumor hypoxia with MRI in vivo. 


\section{MATERIALS AND METHODS}

\section{Phantom validation of $R_{1}$ measurement}

In vitro validation experiments were performed on the same 7T system as used for the in vivo studies. The phantom consisted of four $5 \mathrm{~mm}$ NMR tubes (Sigma- Aldrich, UK) with solutions of gadopentetate dimeglumine (Magnevist; Schering, Berlin, Germany) serially diluted in water to yield final concentrations of $0.01,0.05,0.07$ and $0.09 \mathrm{mM}$ gadolinium and an expected range of $T_{1}$ between 1400-2500ms based on the relaxivity of Magnevist at $7 \mathrm{~T}$ at $37^{\circ} \mathrm{C}(27)$. NMR tubes were placed inside a plastic container filled with dental paste to reduce susceptibility effects and to ensure efficient heat transfer for constant temperature. Three repeated measurements of $R_{1}$ were measured for each tube on three separate days.

\section{Tumor implantation}

All experiments were performed in compliance with licenses issued under the UK Animals (Scientific Procedures) Act 1986 and following local ethical review. Studies were compliant with the United Kingdom National Cancer Research Institute guidelines for animal welfare in cancer research (28) and with the ARRIVE guidelines (29).

Three cell lines exhibiting differing vascular and hypoxic phenotypes in vivo were used. Parental 786-0 renal carcinoma cells (ATCC, LCG Standards, Teddington, UK; purchased 2011; 786-0-par), and cells established from a sunitinib refractory subcutaneous 786-0 xenograft (786-0-R) (30), were cultured in RPMI supplemented with $10 \%$ (v/v) fetal calf serum (Gibco, Life Technologies, Paisley, UK). Tumors were propagated by injecting $3 \times 10^{6}$ cells in $100 \mu$ l of sterile PBS into the flanks of 8 week old female C.B17-scid mice under isoflurane anesthesia. SW-620 colorectal carcinoma cells (ATCC; lot \#8924081, purchased 2005) were cultured in DMEM supplemented with $10 \%$ fetal calf serum. Tumors were propagated by injecting $5 \times 10^{6}$ cells in $100 \mu$ of sterile PBS into the flanks of 8 week old female athymic NCr-Foxn $1^{\text {nu }}$ mice. Immediately prior to in vivo implantation, all cells tested negative for mycoplasma infection and the 
number of short tandem repeats (STRs) present at 7-10 loci were assessed by PCR to provide STR profiles, from which cell line authenticity was confirmed.

Tumor size was monitored using callipers and the formula for ellipsoid volume, $(\pi / 6)$ $\times L \times W \times D$, where $L, W$ and $D$ are the largest orthogonal dimensions of the ellipsoid. Tumors were typically used for experimentation at a volume of approximately $400 \mathrm{~mm}^{3}$.

\section{Measurement of tissue $\mathrm{pO}_{2}$}

The fiber optic oxygen sensing device OxyLite ${ }^{\mathrm{TM}}$ (Oxford Optronix, Oxford, UK) (31) was used to measure tumor partial pressure of oxygen $\left(\mathrm{pO}_{2}\right.$; units $\left.\mathrm{mm} \mathrm{Hg}\right)$. A heat mat maintained core body temperature and gas delivery was at $2 \mathrm{l} / \mathrm{min}$ via a nosepiece. Two dual $\mathrm{pO}_{2}$ and temperature probes were positioned within the tumor tissue. Animals initially breathed medical air, followed by $100 \%$ oxygen to mimic the gas changes induced during the MRI experiments. Data acquisition and averaging were performed using Chart version 5 ( $\mathrm{AD}$ Instruments, Castle Hill, Australia). Tissue $\mathrm{pO}_{2}$ data were collected the day after corresponding MRI data.

\section{MRI data acquisition}

Anesthesia was induced with a $10 \mathrm{ml} / \mathrm{kg}$ intraperitoneal injection of fentanyl citrate $(0.315 \mathrm{mg} / \mathrm{ml})$ plus fluanisone $(10 \mathrm{mg} / \mathrm{ml})$ (Hypnorm; Janssen Pharmaceutical Ltd, High Wycombe, UK), midazolam (5mg/ml) (Hypnovel; Roche, Welwyn Garden City, UK) and sterile water (at 1:1:2 ratio) (16). Mice were positioned in a $3 \mathrm{~cm}$ birdcage coil on a custom built platform to isolate the tumor, which was surrounded by dental paste (3M; Bracknell, UK) to minimize motion and susceptibility artifacts. Gas delivery (medical air or $100 \%$ oxygen) was continuous at $21 /$ min through a nose piece. Warm air maintained animal core temperature at $37^{\circ} \mathrm{C}$. Lateral tail vein cannulation was performed with a heparinised 27G butterfly catheter (Venisystems, Hospira, Royal Leamington Spa, UK) to enable the remote intravenous administration of gadolinium contrast agent in DCEMRI studies or when vasomodulator was administered. 
All MRI data were acquired on a 7T horizontal bore microimaging system (Bruker Instruments, Ettlingen, Germany). Localization was performed using a multi-slice turboRARE $\mathrm{T}_{2}$-weighted sequence and was followed by shimming over the tumor (32). All sequences were acquired using $30 \mathrm{~mm} \times 30 \mathrm{~mm}$ FOV (128 $\times 128$ matrix; in plane resolution $0.234 \mathrm{~mm}$ ) for a single $1 \mathrm{~mm}$ thick slice. Experimental protocols are detailed in Supplementary Figure S1. Sequences used were:

OE-MRI: Inversion recovery (IR) True-FISP images were used to calculate $\mathrm{R}_{1}$ (TR $2.4 \mathrm{~ms}$, scan TR 10s, 48 inversion times spaced $38.8 \mathrm{~ms}$ apart with initial inversion time of $106.2 \mathrm{~ms}$; TE $1.2 \mathrm{~ms} ; \alpha 60^{\circ}$ ). High signal-to-noise (SNR) images were used to obtain highly accurate $R_{1}$; these required 8 signal averages and took $10 \mathrm{~min} 40$ s to perform. Dynamic images were used to quantify the temporal onset of $\mathrm{R}_{1}$ changes induced by switching between air and 100\% oxygen. The dynamic images required 2 signal averages and took $2 \mathrm{~min} 40$ s to perform.

BOLD: Multiple gradient echo (MGE) images were used to calculate $\mathrm{R}_{2}{ }^{*}$ (TR 200ms; 8 echo times, TE 6.2 to $28.2 \mathrm{~ms}$ with $3.1 \mathrm{~ms}$ echo spacing; 8 signal averages); duration $3 \min 25 \mathrm{~s}$.

DCE-MRI: Data were collected using a modified True-FISP sequence (TR $2.4 \mathrm{~ms}$, scan TR 10s, 8 inversion times spaced $155 \mathrm{~ms}$ apart, initial inversion time $108 \mathrm{~ms}$, TE $1.2 \mathrm{~ms}$; $\alpha 60^{\circ}$; one signal average, temporal resolution 20s). After five baseline measurements, $0.1 \mathrm{mmol} / \mathrm{kg}$ bolus of the gadolinium-based contrast agent Magnevist $(2 \mathrm{ml} / \mathrm{kg} 50 \mathrm{mM}$ solution) was injected intravenously at $2 \mathrm{ml} / \mathrm{min}$ using a power injector; duration $10 \mathrm{mins}$.

\section{MRI analysis}

Regions of interest (ROIs) were drawn around the tumor on the $\mathrm{T}_{2}$-weighted images by an experienced operator (YJ). ROI were transferred to all OE-MRI, BOLD and DCEMRI data. Voxel-wise and median values of $R_{1}$ and $R_{2}{ }^{*}$ were calculated for each map using a Bayesian maximum a posteriori approach, with in-house software. 
For OE-MRI the voxel wise $\Delta R_{1}$ was calculated, where $\Delta R_{1}=R_{1}\left(O_{2}\right)-R_{1}$ (air). The initial $R_{1}$ (air) was derived from the average of the first two high SNR $R_{1}$ maps. The $R_{1}$ $\left(\mathrm{O}_{2}\right)$ was derived from the high SNR $\mathrm{R}_{1}$ map acquired during oxygen breathing. Oxygen enhancement was measured as $2 \times$ tumor baseline $R_{1} \times$ cohort CoV. Voxels were then classified as enhancing (termed "Oxy-E) or refractory (termed "Oxy-R") to oxygen challenge. Voxel-wise BOLD $\Delta R_{2}{ }^{*}$ was calculated, where $\Delta R_{2}{ }^{*}=R_{2}{ }^{*}\left(O_{2}\right)-R_{2}{ }^{*}$ (air).

Where DCE-MRI was performed, the initial area under the contrast agent concentration curve from 0 to 60 seconds $\left(I A \cup C_{60}\right)$ was calculated (16) and voxels were classified as enhancing when $I A U C_{60}>0$. Where both OE-MRI and DCE-MRI were acquired, data were combined to distinguish three tumor sub-regions from one another: (1) perfused Oxy-E voxels; (2) perfused Oxy-R voxels; and (3) non-perfused voxels.

\section{Hydralazine challenge}

Hydralazine acts directly on vascular smooth muscle in vessels of normal tissues, causing vasodilation and reduced mean arterial blood pressure. Tumor blood vessels lacking smooth muscle do not dilate in response to hydralazine. Hence, blood is redistributed away from the tumor, reducing blood flow and increasing hypoxia within 30 minutes (33-36). Hydralazine challenge has, therefore, been used as a tool to manipulate acute hypoxia.

An initial air-to-oxygen gas challenge was performed. Then gas delivery was switched back to air breathing and intravenous injection of either $5 \mathrm{mg} / \mathrm{kg}$ hydralazine hydrochloride (Sigma-Aldrich Co., Dorset, UK) or saline was performed. Finally, a second air-to-oxygen gas challenge was performed. Spatial differences between the $\Delta R_{1}$ defined Oxy-E and Oxy-R voxels on the two air-to-oxygen challenges were assessed by mismatch mapping. Formal randomisation was not employed, rather during each day's scanning mice were prospectively assigned with intent to balance treatment groups according to tumour size. 


\section{Immunofluorescence analysis}

Intraperitoneal injection of $60 \mathrm{mg} / \mathrm{kg}$ pimonidazole (Hypoxyprobe, Burlington, MA) was performed 55 minutes before $100 \% \quad \mathrm{O}_{2}$ inhalation began to allow for maximal bioreduction of the agent in hypoxic tumor regions. 15mg/kg Hoechst 33342 (SigmaAldrich Co., Dorset, UK) was administered intravenously one minute prior to rapid tumor excision. Tumors were excised whole and bisected along the imaging plane so that the cut surface approximated to the MRI region of interest. Half the tumor was snap frozen and half was formalin fixed and paraffin embedded.

Frozen tissue sections $(5 \mu \mathrm{m})$ were obtained from snap frozen tumor material and scanned using fluorescent microscopy on a Panoramic 250 Flash system (3DHistech, Budapest, Hungary) to determine the number of Hoechst-stained (perfused) vessels (excitation 350nm/emission 480nm). Pimonidazole binding was determined in the same sections using Hypoxyprobe-1 (Hypoxyprobe, Burlington, MA), a mouse-monoclonal, followed by rabbit anti-mouse-fluorescein isothiocyanate (FITC) conjugated secondary antibody (excitation $488 \mathrm{~nm} /$ emission $525 \mathrm{~nm}$ ). Paraffin embedded sections $(5 \mu \mathrm{m})$ were obtained from tumor tissue dehydrated after fixation in 10\% phosphate-buffered formalin. Sections were stained with hematoxylin and eosin (H\&E). Data was analyzed using ImageJ software ( $\mathrm{NIH}$, Bethesda, MD). The perfused vessel area, hypoxic fraction and percentage necrosis were calculated, as described elsewhere (37).

\section{Statistical analysis}

IBM SPSS Statistics v.22 (Armonk, NY) was used for all statistical analysis. In all cases, $p$ values of $<0.05$ were considered significant. Comparison of median values of $\mathrm{R}_{1}$ during medical air only breathing and during oxygen challenge experiments were evaluated using a one way analysis of variance (ANOVA) with a post hoc Bonferroni correction for multiple comparisons.

Data comparing median values of $\mathrm{R}_{1}, \mathrm{R}_{2}{ }^{*}$ and $I A U C_{60}$ and fractions of Oxy-E or Oxy-R between different tumor cohorts (786-0-R, 786-0-par or SW620) were evaluated with independent t-tests corrected for multiple comparisons. Data for different cohorts were 
Author Manuscript Published OnlineFirst on December 9, 2015; DOI: 10.1158/0008-5472.CAN-15-2062

Author manuscripts have been peer reviewed and accepted for publication but have not yet been edited.

assumed to be distributed normally and have unequal variance. Correlations between $\mathrm{MRI}$ and pathology were assessed by the non-parametric Spearman's rho. Formal sample size calculations were not performed. 


\section{RESULTS}

\section{OE-MRI signals are accurate, precise, stable and sensitive to $\mathrm{pO}_{2}$ change}

We sought to demonstrate OE-MRI signal precision, stability and sensitivity to altered oxygen tension, because these factors have not been well documented. Since OE-MRI signal changes are based on the longitudinal relaxation rate, $R_{1}$, we compared the expected $R_{1}$ against the measured $R_{1}$ in an in vitro phantom. These data showed that our $\mathrm{R}_{1}$ measurement technique is accurate (Supplementary Figure $\mathrm{S} 2$ ).

To test measurement precision and stability in vivo, we then performed an experiment in four mice implanted with subcutaneous $786-0-R$ tumors $(786-0-R$ is a fast-growing subline of the 786-0 renal cancer cell line). Mice initially breathed medical air for 40 minutes. This was followed by $100 \%$ oxygen challenge for 20 minutes. Finally the mice breathed medical air again for 25 minutes. Multiple $R_{1}$ maps were acquired (Supplementary Figure S1a). The within-scan co-efficient of variation (CoV) of baseline voxel-wise $\mathrm{R}_{1}$ was $0.41 \%$ in vivo, indicating high measurement precision. No appreciable difference was observed in the spatial mapping of tumor $\mathrm{R}_{1}$ during air breathing (see time points 1-7 in Figure 2a). No significant change was seen in tumor $R_{1}$ in the four mice (Figure $2 b$ ), indicating signal stability while breathing air.

Challenge with $100 \%$ oxygen was then performed in the same mice, to increase tumor $\mathrm{pO}_{2}$. All four tumors showed rapid, heterogeneous and significant increase in median $\mathrm{R}_{1}$ following oxygen challenge. These changes were clearly visible in the first map after $2 \mathrm{~min} 40$ s of oxygen breathing and persisted for the duration of the oxygen challenge ( $p<0.008$ for all time points 8-12 compared with air breathing) showing consistent spatial arrangement (Figure 2a-b). Across all four tumors, $89.3 \%$ of voxels were oxygen enhancing (Oxy-E) and $10.7 \%$ of voxels were oxygen refractory (Oxy-R). Sample traces of $\Delta R_{1}$ change are shown for Oxy-E and Oxy-R voxels (Supplementary Figure S3). OxyLite ${ }^{\mathrm{TM}}$ measurement in the same tumors showed $\mathrm{pO}_{2}$ increase (beyond the limit of detection of the OxyLite ${ }^{\mathrm{TM}}$ equipment, namely at 100mmHg) in 7 out of 8 measured regions over the same time frame (sample trace in Figure 2c). The OxyLite ${ }^{\mathrm{TM}}$ data 
provides independent evidence that OE-MRI detects real time increases in the oxygen concentration in tumor interstitial tissue fluid. BOLD MRI, performed in the same mice, revealed a significant reduction in $\mathrm{R}_{2}{ }^{*}$ during oxygen challenge (mean reduction 39.8 $\mathrm{ms}^{-1}$; SE $\left.11.7 \mathrm{~ms}^{-1} ; \mathrm{p}=0.043\right)$, consistent with previous studies (38).

Tumors showed rapid reversal of $R_{1}$ changes when oxygen challenge ended and air breathing was resumed (see time points 13-17 in Figure 2a). There was no significant difference between baseline and end-of-study mean $\mathrm{R}_{1}$ values for the cohort. OxyLite ${ }^{\mathrm{TM}}$ measurement showed $\mathrm{pO}_{2}$ return to pre-challenge levels over a period of 2 to 6 minutes mirroring $\Delta R_{1}$ change. Collectively these data confirm that $R_{1}$ measurements used for this study are accurate, precise, stable in the absence of perturbation and sensitive to change in tumour $\mathrm{pO}_{2}$.

\section{Oxy-R fraction detects differential levels of hypoxia in an isogenic system}

We investigated whether Oxy-R fraction (the fraction of each tumor refractory to oxygen challenge) could detect different levels of hypoxia in cancer models. We measured the Oxy-R fraction in two isogenic cell lines: the slow growing parental 786-0 cell line (7860-par), which took 206.3 days $\pm 66.2 \mathrm{SD}$ to reach a tumor volume of $\sim 400 \mathrm{~mm}^{3}$ ( $\mathrm{n}=8$ mice), and the much faster growing $786-0$ subline (786-0-R), which only took only 32.1 days $\pm 6.9 \mathrm{SD}$ to reach the same tumor volume $(\mathrm{n}=9$ mice). From these mice we acquired OE-MRI, $\Delta \mathrm{R}_{2}{ }^{*}$ and DCE-MRI data along with histopathological analysis of pimonidazole adduct formation (for hypoxia) and Hoechst 33342 uptake (for perfusion) (Supplementary Figure S1b).

Staining for pimonidazole adduct formation showed a significantly higher hypoxic fraction in 786-0-R tumors compared to the 786-0-par tumors $(p=0.008)$ (Figure 3a). This finding was mirrored by the Oxy-R fraction being significantly higher in the 786-0-R xenografts compared with the 786-0-par xenografts $(p=0.047)$ (Figure 3b). In distinction, there was no significant difference in the median values of $\Delta R_{1}, I A U C_{60}$ or $\Delta \mathrm{R}_{2}{ }^{*}$ between the 786-0- $\mathrm{R}$ and 786-0-par xenografts (Figure 3c-e). No significance difference was seen in the perfused vessel area measured by Hoechst 33342 staining 
(data not shown). These data confirm that $O x y-R$ fraction is sensitive to differential levels of hypoxia but that median values of $\Delta R_{1}, I A U C_{60}$ and $\Delta R_{2}{ }^{*}$ were insensitive to this difference.

\section{Perfused Oxy-R quantifies hypoxic fraction}

We sought to test if Oxy-R fraction could estimate tumor hypoxic fraction in two tumor models originating from different cell lines. In particular, we wanted to test if the presence of avascular or necrotic tumor tissue - which does not receive any delivery of oxygen gas in the blood plasma - would confound the relationship between Oxy-R and hypoxia. Therefore we analyzed data from the perfused portion of each tumor.

To do this, we first used the 786-0-R data already collected. We examined the within cohort correlation between $\mathrm{Oxy}-\mathrm{R}$ fraction and the pimonidazole adduct formation based measurement of hypoxic fraction. This model is known to be highly vascularized. Further, there was a wide dynamic range of hypoxia in this model. We then performed an equivalent experiment using SW620 tumors. This model was chosen because these tumors are relatively poorly vascularized (39).

Anticipated differences in the perfusion status of the two models were confirmed; 786$0-R$ xenografts had significantly greater perfused tumor area compared with SW620 xenografts, measured by DCE-MRI $(p=0.011)$ (Table 1) and by Hoechst 33342 $(p=0.004)$. Pathology analysis showed that perfused vessel area measured by Hoechst 33342 (Supplementary Figure S4a-b) correlated with perfused Oxy-E fraction (those voxels showing positive enhancement with both oxygen and gadolinium) and that nonperfused fraction correlated with necrosis on H\&E in both models (Supplementary Figure S4c-d).

Oxy-R fraction correlated strongly with pathological hypoxic fraction in 786-0-R tumours (rho $0.810, p=0.028$ ) (Figure $4 a$ ), irrespective of whether the entire tumor was analyzed or if analysis was restricted to gadolinium-enhancing tumor only (perfused Oxy- $R$ fraction). In SW620 tumours the Oxy-R fraction did not correlate with hypoxic fraction 
(measured by pimonidazole adduct formation). However, as anticipated the perfused Oxy-R fraction and hypoxic fraction (measured by pimonidazole adduct formation) correlated strongly (rho 0.929, $p=0.002$ ) (Figure 4b). In both models, visual inspection revealed that perfused $O x y-R$ voxels were located at an interface between the peripheral perfused Oxy-E voxels and the centrally located non-perfused voxels. Collectively, these data provide evidence that Oxy-R fraction can quantify hypoxic fraction accurately, but requires perfusion data in poorly vascularized tumors.

\section{Oxy-R fraction is sensitive to dynamic change in hypoxia}

In order to provide evidence that Oxy-R fraction can detect reduced oxygen delivery to tumors, we administered the vasomodulator hydralazine to a cohort of mice bearing 786-0-R xenografts ( $n=4$ mice) and compared with control mice receiving saline $(n=6$ mice). We performed an air-to-oxygen challenge before and after the administration of hydralazine or control (Supplementary Figure S1c). In the initial air-to-oxygen challenge, overall $\Delta R_{1}$ changes were positive, as expected in all ten mice (Figure 5a).

Importantly, the second air-to-oxygen challenge was initiated 15 minutes after administration of hydralazine or saline. The magnitude of $\Delta R_{1}$ was reduced significantly in mice receiving hydralazine, relative to control (Figure 5a) $(p<0.01)$. This difference in hydralazine-treated tumors was driven by the Oxy-R fraction increasing significantly from $17.3 \%$ to $30.5 \%(p=0.045)$ (Figure $5 b)$.

To further understand these changes, we used 'mismatch mapping' to identify tumor sub-regions that changed oxygen enhancement status. In control tumors, the majority of voxels remained consistently either Oxy-E or Oxy-R. The number of voxels changing from Oxy-E to Oxy-R or vice versa was equal for both directions of change and was small (Figure 5c). In hydralazine treated tumors, substantially more voxels turned from Oxy-E to Oxy-R than vice versa. Some of these newly Oxy-R tumor sub-regions were immediately adjacent to established Oxy-R regions, but other new areas of Oxy-R were detected at spatially distant sub-regions (Figure $5 \mathrm{~d}$ ). These data confirm that OE-MRI 
Author Manuscript Published OnlineFirst on December 9, 2015; DOI: 10.1158/0008-5472.CAN-15-2062

Author manuscripts have been peer reviewed and accepted for publication but have not yet been edited.

can detect dynamic change in tumor hypoxia. Further, tumor regions with appearing or resolving hypoxia can be mapped using this technique. Research. 


\section{DISCUSSION}

Hypoxia is an important prognostic factor for solid tumors and mediates resistance to radiotherapy and to some chemotherapy agents. Non-invasive clinical imaging has potential to improve patient care by producing biomarkers that alter clinical decision making $(40,41)$. In the case of hypoxia, a clinically translational imaging biomarker has potential to be prognostic and/or predictive (identifying presence of hypoxia and quantifying hypoxic fraction/volume prior to therapy), to assist treatment planning (mapping targets for radiation boost or adaptive therapy) (42), or to monitor therapy induced changes in tumor hypoxia, for example following radiosensitisation or hypoxia activated cytotoxic therapy $(10,43)$.

To date, clinical strategies to image tumor hypoxia have been dominated by positron emission tomography (PET) methods (7). Several investigational PET tracers have been evaluated, each with slightly different underlying relationship to tissue oxygen tension $(44,45)$. However, at present no imaging method identifies and maps the extent of tumor hypoxia that is validated, cost-effective, widely available and used for patient benefit (7).

OE-MRI is an emerging technique that detects the presence of excess dissolved $\mathrm{O}_{2}$ molecules in plasma and tissue fluid. Most OE-MRI studies in cancer have been small cohort based studies, and without corresponding pathology or intervention, tending to report changes in median signal intensity or median $\Delta R_{1}$. In well oxygenated tumor tissue, oxygen-induced $\Delta R_{1}$ signals are positive, similar to signal changes observed in healthy tissues $(46,47)$. Advances in OE-MRI have focused largely around providing dual $\Delta R_{1}$ and $\Delta R_{2}{ }^{*}$ acquisitions (25) or in enhancing the magnitude of oxygen-induced $\Delta R_{1}$, for example by developing methods sensitive to tumor lipid signals (17).

In this study, we adopted a different approach. We focused on the fact that tumors are biologically heterogeneous, composed of sub-regions with distinct pathophysiology (48, 49). Previous OE-MRI studies have suggested that some tumor sub-regions are 
refractory to the positive $\Delta \mathrm{R}_{1}$ induced by oxygen-challenge $(14,20)$. We built on this observation by partitioning the OE-MRI signal into regions with enhancing (Oxy-E) or refractory (Oxy-R) signatures. We conducted a series of experiments to test the hypothesis that Oxy-R fraction tumor can identify, quantify and map tumor hypoxia.

We first used the renal carcinoma model 786-0-R (rapid growing, vascular tumors with anticipated large oxygen-induced $\Delta R_{1}$ ) to develop a protocol that was not only stable while breathing medical air, but also sensitive to oxygen-induced signals. This is the first study to provide detailed evidence of measurement stability and sensitivity for serial $R_{1}$ mapping in OE-MRI. The temporal evolution of oxygen-enhanced positive $\Delta R_{1}$ occurred within 3 minutes and was spatially stable. The OE-MRI data mirrored OxyLite ${ }^{\mathrm{TM}}$ measurement of $\mathrm{pO}_{2}$ changes, concurring with data from a previous study where rapid increases in $\Delta R_{1}$ were observed following gas challenge (15) and with data comparing OE-MRI signal changes with ${ }^{19} \mathrm{~F}$ oximetry (19).

Next, we demonstrated that Oxy-R fraction could distinguish differences in hypoxic fraction within tumors derived from two isogenic cell lines: parental 786-0 cells and 786$0-R$ cells. Here, the relatively rapid growing 786-0-R tumors had significantly more hypoxia than the slow growing 786-0-par tumors. Crucially, Oxy-R fraction distinguished between cell lines, mirroring differences observed in values of hypoxic fraction quantified through pimonidazole staining.

We then tested if Oxy-R fraction could estimate hypoxic fraction. We chose the 786-0-R and SW620 models because of their differing perfusion characteristics. Significant correlation was seen between Oxy-R fraction and hypoxic fraction (defined on pimonidazole adduct formation) in the relatively well perfused $786-0-R$ xenografts. This association was not replicated in the poorly perfused SW620 xenografts because many Oxy-R voxels had negligible perfusion, so received insufficient oxygen gas to alter voxel $R_{1}$. Having accounted for these voxels using DCE-MRI, we measured Oxy-R fraction in perfused tissue and observed a significant association in the SW620 tumors. 
We then increased the fraction of tumor hypoxia in a further cohort of 786-0-R tumors using the vasomodulator hydralazine. Oxy-R fraction increased two-fold, providing direct evidence that OE-MRI can track acute changes in tumor oxygenation. Some signal fluctuation was observed in both directions in hydralazine and control tumors. This may reflect changes in acute transient hypoxia (50), but further work is required to understand these signal changes.

In these experiments, median $\Delta \mathrm{R}_{1}$ (the most common biomarker derived from OE-MRI data) was unable to distinguish the differing levels of hypoxia between the 786-0 sublines and did not correlate with the degree of hypoxic fraction within the SW620 tumors. We also compared the Oxy-R analysis approach with BOLD MRI. Oxygen-induced $\Delta \mathrm{R}_{2}{ }^{*}$ did mirror changes in tissue $\mathrm{pO}_{2}$, similar to previous reports (38), but $\Delta \mathrm{R}_{2}{ }^{*}$ did not differ between the 786-0 sub-lines with differing levels of hypoxia, nor did $\Delta \mathrm{R}_{2}{ }^{*}$ relate to degree of hypoxic fraction in 786-0-R or SW620 tumors, suggesting that $R_{2}$ * measurements were confounded by other factors, such as vessel flow. These findings are consistent with previous reports of inconsistent relationships between $\Delta R_{2}{ }^{*}$ and pimonidazole-based assay of hypoxic fraction (38).

Our findings are important for at least four reasons. Firstly, these data demonstrate that positive $\Delta \mathrm{R}_{1}$ changes on $\mathrm{OE}-\mathrm{MRI}$ imaging are sufficiently precise, stable and strong enough to be measured reliably. This enables those portions of tumor refractory to positive signal change (i.e. Oxy-R sub-regions) to be identified with confidence. Secondly, these data suggest that in OE-MRI, Oxy-R fraction relates more closely to tumor hypoxia than median $\Delta R_{1}$. This supports further evaluation of Oxy-R fraction or Oxy-R tumor volume as a prognostic and/or predictive biomarker. Thirdly, Oxy-R voxels were located in spatially distinct sub-regions suggesting that, if replicated in humans, this technique may facilitate the planning of localized boost and adaptive radiotherapy delivery strategies. Fourthly, clinical tumors vary in their extent of perfusion and this may further change with therapy. Our data suggest that subsequent studies of OE-MRI in patients with cancer should evaluate Oxy-R fraction in perfused tumor regions (using 
a combined analysis with DCE-MRI or an equivalent technique), rather than using OE$\mathrm{MRI}$ in isolation.

In conclusion, these data provide substantial evidence to validate the measurement of Oxy-R fraction to identify, quantify and map tumor hypoxia. Unlike other nuclear magnetic resonance techniques such as electron paramagnetic resonance oximetry, ${ }^{19} \mathrm{~F}$ oximetry and Overhauser enhanced MRI - which are either invasive or require use of an investigational diagnostic agent, hindering clinical translation (7) - Oxy-R fraction can be readily quantified on clinical MRI scanners (24), making the technique suitable for rapid clinical translation.

Acknowledgements: We thank Allan Thornhill and his staff (ICR) for animal maintenance and Dr Damian McHugh (University of Manchester) for generation of maps used in the figures. 


\section{REFERENCES}

1. Harris AL. Hypoxia--a key regulatory factor in tumour growth. Nat Rev Cancer 2002; 2:38-47.

2. Pugh CW, Ratcliffe PJ. Regulation of angiogenesis by hypoxia: role of the HIF system. Nat Med 2003; 9:677-684.

3. Hockel M, Schlenger K, Aral B, Mitze M, Schaffer U, Vaupel P. Association between tumor hypoxia and malignant progression in advanced cancer of the uterine cervix. Cancer Res 1996; 56:4509-4515.

4. Brizel DM, Sibley GS, Prosnitz LR, Scher RL, Dewhirst MW. Tumor hypoxia adversely affects the prognosis of carcinoma of the head and neck. Int J Radiat Oncol Biol Phys 1997; 38:285-289.

5. Vergis R, Corbishley CM, Norman AR, Bartlett J, Jhavar S, Borre M, et al. Intrinsic markers of tumour hypoxia and angiogenesis in localised prostate cancer and outcome of radical treatment: a retrospective analysis of two randomised radiotherapy trials and one surgical cohort study. Lancet Oncol 2008; 9:342-351.

6. Wilson WR, Hay MP. Targeting hypoxia in cancer therapy. Nat Rev Cancer 2011; 11:393-410.

7. Tatum JL, Kelloff GJ, Gillies RJ, Arbeit JM, Brown JM, Chao KS, et al. Hypoxia: importance in tumor biology, noninvasive measurement by imaging, and value of its measurement in the management of cancer therapy. Int J Radiat Biol 2006; 82:699-757.

8. O'Connor JP, Jackson A, Parker GJ, Roberts C, Jayson GC. Dynamic contrastenhanced MRI in clinical trials of antivascular therapies. Nat Rev Clin Oncol 2012; 9:167-177.

9. Howe FA, Robinson SP, Rodrigues LM, Griffiths JR. Flow and oxygenation dependent (FLOOD) contrast MR imaging to monitor the response of rat tumors to carbogen breathing. Magn Reson Imaging 1999; 17:1307-1318.

10. Hammond EM, Asselin MC, Forster D, O'Connor JP, Senra JM, Williams KJ. The meaning, measurement and modification of hypoxia in the laboratory and the clinic. Clin Oncol (R Coll Radiol) 2014; 26:277-288.

11. Young IR, Clarke GJ, Bailes DR, Pennock JM, Doyle FH, Bydder GM. Enhancement of relaxation rate with paramagnetic contrast agents in NMR imaging. J Comput Tomogr 1981; 5:543-547.

12. Berkowitz BA. Role of dissolved plasma oxygen in hyperoxia-induced contrast. Magn Reson Imaging 1997; 15:123-126.

13. Gray LH, Steadman JM. Determination of the Oxyhaemoglobin Dissociation Curves for Mouse and Rat Blood. J Physiol 1964; 175:161-171.

14. Matsumoto K, Bernardo M, Subramanian S, Choyke P, Mitchell JB, Krishna MC, et al. MR assessment of changes of tumor in response to hyperbaric oxygen treatment. Magn Reson Med 2006; 56:240-246.

15. Winter JD, Akens MK, Cheng HL. Quantitative MRI assessment of VX2 tumour oxygenation changes in response to hyperoxia and hypercapnia. Phys Med Biol 2011; 56:1225-1242.

16. Burrell JS, Walker-Samuel S, Baker LC, Boult JK, Jamin Y, Halliday J, et al. Exploring DeltaR(2) * and DeltaR(1) as imaging biomarkers of tumor oxygenation. J Magn Reson Imaging 2013; 38:429-434.

17. Jordan BF, Magat J, Colliez F, Ozel E, Fruytier AC, Marchand V, et al. Mapping of oxygen by imaging lipids relaxation enhancement: A potential sensitive endogenous MRI contrast to map variations in tissue oxygenation. Magn Reson Med 2013; 70:732744. 
18. Colliez F, Neveu MA, Magat J, Cao Pham TT, Gallez B, Jordan BF. Qualification of a noninvasive magnetic resonance imaging biomarker to assess tumor oxygenation. Clin Cancer Res 2014; 20:5403-5411.

19. Hallac RR, Zhou H, Pidikiti R, Song K, Stojadinovic S, Zhao D, et al. Correlations of noninvasive BOLD and TOLD MRI with $\mathrm{pO} 2$ and relevance to tumor radiation response. Magn Reson Med 2014; 71:1863-1873.

20. Linnik IV, Scott ML, Holliday KF, Woodhouse N, Waterton JC, O'Connor JP, et al. Noninvasive tumor hypoxia measurement using magnetic resonance imaging in murine U87 glioma xenografts and in patients with glioblastoma. Magn Reson Med 2014; 71:1854-1862.

21. Zhao D, Pacheco-Torres J, Hallac RR, White D, Peschke P, Cerdan S, et al. Dynamic oxygen challenge evaluated by NMR T1 and T2 * - insights into tumor oxygenation. NMR Biomed 2015; 28:937-947.

22. Beeman SC, Shui YB, Perez-Torres CJ, Engelbach JA, Ackerman JJ, Garbow JR. O2 sensitive MRI distinguishes brain tumor versus radiation necrosis in murine models. Magn Reson Med 2015:Published OnlineFirst July 14, 2015.

23. Arnold JF, Kotas M, Fidler F, Pracht ED, Flentje M, Jakob PM. Quantitative regional oxygen transfer imaging of the human lung. J Magn Reson Imaging 2007; 26:637-645.

24. O'Connor JP, Naish JH, Parker GJ, Waterton JC, Watson Y, Jayson GC, et al. Preliminary study of oxygen-enhanced longitudinal relaxation in MRI: a potential novel biomarker of oxygenation changes in solid tumors. Int J Radiat Oncol Biol Phys 2009; 75:1209-1215.

25. Remmele S, Sprinkart AM, Muller A, Traber F, von Lehe M, Gieseke J, et al. Dynamic and simultaneous MR measurement of R1 and R2* changes during respiratory challenges for the assessment of blood and tissue oxygenation. Magn Reson Med 2013; 70:136-146.

26. Blockley NP, Jiang L, Gardener AG, Ludman CN, Francis ST, Gowland PA. Field strength dependence of $\mathrm{R} 1$ and $\mathrm{R} 2^{*}$ relaxivities of human whole blood to ProHance, Vasovist, and deoxyhemoglobin. Magn Reson Med 2008; 60:1313-1320.

27. Kalavagunta C, Michaeli S, Metzger GJ. In vitro Gd-DTPA relaxometry studies in oxygenated venous human blood and aqueous solution at 3 and $7 \mathrm{~T}$. Contrast Media Mol Imaging 2014; 9:169-176.

28. Workman P, Aboagye EO, Balkwill F, Balmain A, Bruder G, Chaplin DJ, et al. Guidelines for the welfare and use of animals in cancer research. $\mathrm{Br} \mathrm{J}$ Cancer 2010; 102:1555-1577.

29. Kilkenny C, Browne WJ, Cuthill IC, Emerson M, Altman DG. Improving bioscience research reporting: the ARRIVE guidelines for reporting animal research. PLoS Biol 2010; 8:e1000412.

30. Bridgeman VL, Wan E, Foo S, Nathan MR, Welti JC, Frentzas S, et al. Preclinical evidence that trametinib enhances the response to anti-angiogenic tyrosine kinase inhibitors in renal cell carcinoma. Mol Cancer Ther 2015:Published OnlineFirst October 20, 2015.

31. Griffiths JR, Robinson SP. The OxyLite: a fibre-optic oxygen sensor. Br J Radiol 1999; 72:627-630.

32. Gruetter R. Automatic, localized in vivo adjustment of all first- and second-order shim coils. Magn Reson Med 1993; 29:804-811.

33. Stratford IJ, Adams GE, Godden J, Nolan J, Howells N, Timpson N. Potentiation of the anti-tumour effect of melphalan by the vasoactive agent, hydralazine. Br J Cancer 1988; 58:122-127. 
34. Horsman MR, Nordsmark M, Hoyer M, Overgaard J. Direct evidence that hydralazine can induce hypoxia in both transplanted and spontaneous murine tumours. $\mathrm{Br} \mathrm{J}$ Cancer 1995; 72:1474-1478.

35. Seddon BM, Maxwell RJ, Honess DJ, Grimshaw R, Raynaud F, Tozer GM, et al. Validation of the fluorinated 2-nitroimidazole SR-4554 as a noninvasive hypoxia marker detected by magnetic resonance spectroscopy. Clin Cancer Res 2002; 8:2323-2335.

36. Zhao D, Constantinescu A, Jiang L, Hahn EW, Mason RP. Prognostic radiology: quantitative assessment of tumor oxygen dynamics by MRI. Am J Clin Oncol 2001; 24:462-466.

37. Williams KJ, Telfer BA, Shannon AM, Babur M, Stratford IJ, Wedge SR. Combining radiotherapy with AZD2171, a potent inhibitor of vascular endothelial growth factor signaling: pathophysiologic effects and therapeutic benefit. Mol Cancer Ther 2007; 6:599-606.

38. Baker LC, Boult JK, Jamin Y, Gilmour LD, Walker-Samuel S, Burrell JS, et al. Evaluation and immunohistochemical qualification of carbogen-induced DeltaR(2) as a noninvasive imaging biomarker of improved tumor oxygenation. Int J Radiat Oncol Biol Phys 2013; 87:160-167.

39. Li J, Jamin Y, Boult JK, Cummings C, Waterton JC, Ulloa J, et al. Tumour biomechanical response to the vascular disrupting agent ZD6126 in vivo assessed by magnetic resonance elastography. Br J Cancer 2014; 110:1727-1732.

40. Parkinson DR, Johnson BE, Sledge GW. Making personalized cancer medicine a reality: challenges and opportunities in the development of biomarkers and companion diagnostics. Clin Cancer Res 2012; 18:619-624.

41. Waterton JC, Pylkkanen L. Qualification of Imaging Biomarkers for Oncology Drug Development. Eur J Cancer 2012; 48:409-415.

42. Gatenby RA, Silva AS, Gillies RJ, Frieden BR. Adaptive therapy. Cancer Res 2009; 69:4894-4903.

43. Ahn GO, Brown M. Targeting tumors with hypoxia-activated cytotoxins. Front Biosci 2007; 12:3483-3501.

44. Carlin S, Zhang H, Reese M, Ramos NN, Chen Q, Ricketts SA. A comparison of the imaging characteristics and microregional distribution of 4 hypoxia PET tracers. J Nucl Med 2014; 55:515-521.

45. Peeters SG, Zegers CM, Lieuwes NG, van Elmpt W, Eriksson J, van Dongen GA, et al. A comparative study of the hypoxia PET tracers [(1)(8)F]HX4, [(1)(8)F]FAZA, and [(1)(8)F]FMISO in a preclinical tumor model. Int J Radiat Oncol Biol Phys 2015; 91:351359.

46. O'Connor JPB, Jackson A, Buonaccorsi GA, Buckley DL, Roberts C, Watson Y, et al. Organ-specific effects of oxygen and carbogen gas inhalation on tissue longitudinal relaxation times. Magn Reson Med 2007; 58:490-496.

47. Winter JD, Estrada M, Cheng HL. Normal tissue quantitative T1 and T2* MRI relaxation time responses to hypercapnic and hyperoxic gases. Acad Radiol 2011; 18:1159-1167.

48. Junttila MR, de Sauvage FJ. Influence of tumour micro-environment heterogeneity on therapeutic response. Nature 2013; 501:346-354.

49. O'Connor JP, Rose CJ, Waterton JC, Carano RA, Parker GJ, Jackson A. Imaging intratumor heterogeneity: role in therapy response, resistance, and clinical outcome. Clin Cancer Res 2015; 21:249-257.

50. Baudelet C, Cron GO, Ansiaux R, Crokart N, Dewever J, Feron O, et al. The role of vessel maturation and vessel functionality in spontaneous fluctuations of $T(2)^{*}$-weighted GRE signal within tumors. NMR Biomed 2006; 19:69-76. 


\section{TABLE}

\section{Table 1}

Proportions of Oxy-E and Oxy-R tumor (with standard deviation; SD), subdivided by voxel perfusion status

\begin{tabular}{|l|l|l|l|l|}
\hline \multirow{2}{*}{} & \multicolumn{2}{|c|}{ \% Oxy-E voxels } & \multicolumn{2}{c|}{ \% Oxy-R voxels } \\
\cline { 2 - 5 } & Perfused & Not perfused & Perfused & Not perfused \\
\hline 786-O-R & 78.2 & 7.2 & 13.2 & 1.2 \\
& (SD18.3) & (SD 5.9) & (SD 12.9) & (SD 1.6) \\
\hline Parental 786-O & 84.7 & 11.0 & 3.4 & 0.9 \\
& (SD 14.0) & (SD 12.2) & (SD 2.5) & (SD 0.8) \\
\hline SW620 & 51.5 & 25.2 & 15.7 & 7.6 \\
& (SD 13.8) & (SD 14.9) & (SD 6.2) & (SD 4.3) \\
\hline
\end{tabular}

\section{FIGURE LEGENDS}

\section{Figure 1}

OE-MRI distinguishes between normoxic and hypoxic tissue. Each box represents an imaging voxel which contains erythrocytes (red spheres) in blood vessels (pink cylinders); tumor cells (gray ellipse); and surrounding interstitial space (white box). A. In normoxic tissue, hemoglobin molecules in erythrocytes are oxygen saturated and form oxyhemoglobin $\left(\mathrm{HbO}_{2}\right)$ molecules. Dioxygen molecules $\left(\mathrm{O}_{2}\right)$ are dissolved in the plasma. Inhalation of hyperoxic gas markedly increases the amount of dissolved plasma $\mathrm{O}_{2}$ but $\mathrm{HbO}_{2}$ concentration is essentially unaltered. B. Increased $\mathrm{pO}_{2}$ in interstitial fluid and plasma increases tissue longitudinal relaxation rate $\left(R_{1}\right)$, which is detected by MRI. C. In hypoxic tissue, hemoglobin molecules are not fully oxygen saturated and exist as deoxyhemoglobin $(\mathrm{Hb})$ molecules. Inhalation of hyperoxic gas increases the $\mathrm{HbO}_{2}$ to $\mathrm{Hb}$ ratio but has negligible effect on plasma $\mathrm{O}_{2}$. D. Since there is negligible change in $\mathrm{pO}_{2}$ the $\mathrm{R}_{1}$ remains little changed (straight black line). Since $\mathrm{Hb}$ has a slightly higher longitudinal relaxivity than $\mathrm{HbO}_{2}$, tissue $\mathrm{R}_{1}$ may even decrease (dotted black line). 


\section{Figure 2}

OE-MRI signals are precise, stable and sensitive to $\mathbf{p O}_{2}$ change. $A, R_{1}$ maps in one 786-0-R xenograft tumor while the mouse breathed air (top row), 100\% oxygen (middle row) and back to air (bottom row). B, $\mathrm{R}_{1}$ measurement was stable in four mice with 7860-R xenografts during the initial 45 minutes of air breathing. Symbols: a 10m40s IRTrueFISP acquisition, $\downarrow 2$ m40s acquisition. Rapid and significant changes in median $\mathrm{R}_{1}$ were induced by $100 \%$ oxygen challenge in the same animals and rapid return to baseline values was observed once air breathing resumed. Mean of individual tumor medians are shown \pm 1 s.e.m. C, OE-MRI signal changes mirror the time course of change in tumor $\mathrm{pO}_{2}$ as shown in a sample Oxylite ${ }^{T M}$ trace from one xenograft (maximum to the detection range was $100 \mathrm{mmHg}$ ).

\section{Figure 3}

Oxy-R fraction identifies differential levels of hypoxia. A, 786-0-R xenografts had significantly greater pimonidazole adduct derived hypoxic fraction than 786-0-par xenografts, mirrored in B, Oxy-R fraction tumor. No difference was seen in $\mathbf{C}$, median $\Delta \mathrm{R}_{1}, \mathbf{D}$, median $I A \cup C_{60}$ or $\mathbf{E}$, median $\Delta \mathrm{R}_{2}{ }^{*}$ between the two cell lines. All error bars are 1 s.e.m., ${ }^{*} p<0.05$.

\section{Figure 4}

Perfused Oxy-R fraction measures hypoxic fraction in multiple tumor models. Representative OE-MRI and DCE-MRI maps from A, 786-0-R and B, SW620 tumors are binarized to show enhancing and non-enhancing voxels. For combined MRI data the voxels are color coded as perfused Oxy-E (yellow), perfused Oxy-R (blue) and nonperfused (gray). Immunofluoresence was performed to map tumor hypoxia by pimonidazole adduct formation. In 786-0-R tumors Oxy-R fraction correlated with hypoxic fraction (and this relationship was maintained when only perfused voxels were assessed). In SW620 tumors, Oxy-R fraction did not correlate significantly with hypoxic fraction, which it overestimated. However when analysis is restricted to perfused Oxy-R voxels (blue squares) a significant correlation was observed. 


\section{Figure 5}

Oxy-R detects reduced oxygen delivery induced by hydralazine. A, Time course of the $\Delta R_{1}$ in $786-0-R$ xenografts challenged with $100 \%$ oxygen, before and after intravenous injection of either saline control or $5 \mathrm{mg} / \mathrm{kg}$ hydralazine hydrochloride. Mean of initial two air breathing $R_{1}$ values taken as baseline for subsequent $\Delta R_{1}$. All error bars are 1 s.e.m. B, Hydralazine-treated tumors showed increase in the Oxy-R fraction with the second oxygen challenge, relative to control. Oxy-E (white) and Oxy-R (black) voxels are mapped for each oxygen challenge in example control and hydralazine treated tumors. C, Mismatch mapping reveals that approximately equal numbers of voxels switch from Oxy-R to Oxy-E (red) and from Oxy-E to Oxy-R (green) in the control tumor. Most voxels remain either Oxy-E (white) or Oxy-R (black) throughout. D, In distinction, Oxy-R fraction increases on the second oxygen challenge with hydralazine due to substantially more voxels switching from Oxy-R to Oxy-E (red) than from Oxy-E to Oxy-R (green). 


\section{FIGURE $1 \quad$ Normoxia}
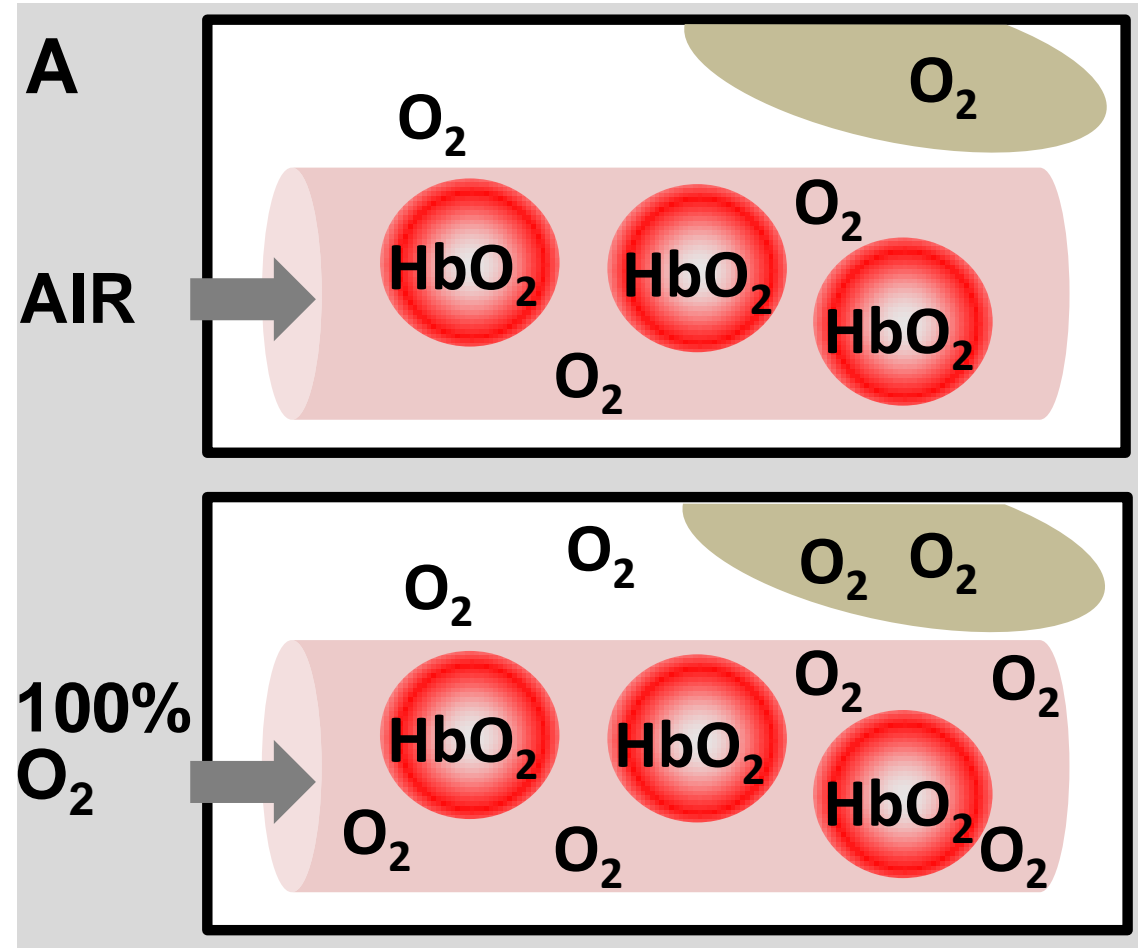

B

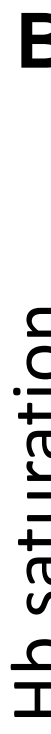

Hypoxia
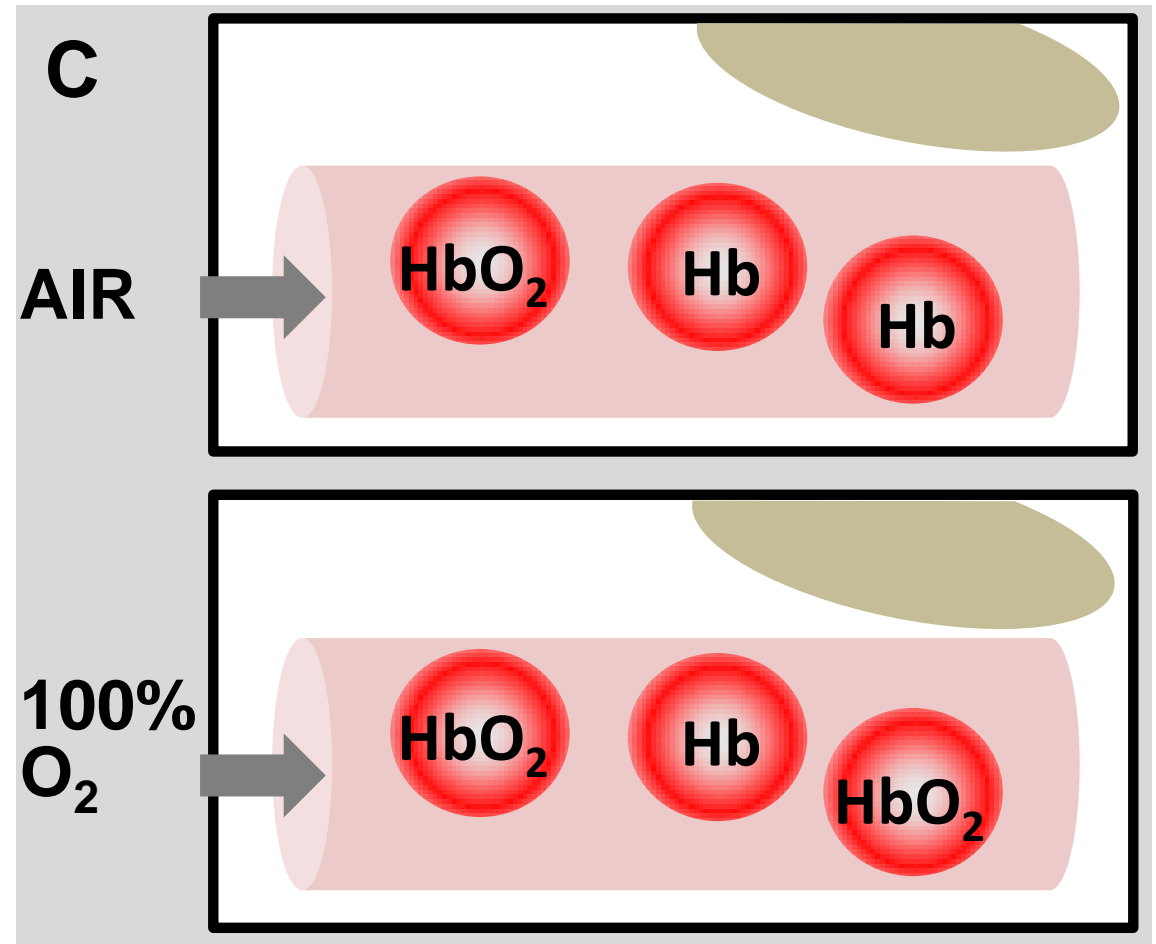

D $\quad R_{1} \uparrow$

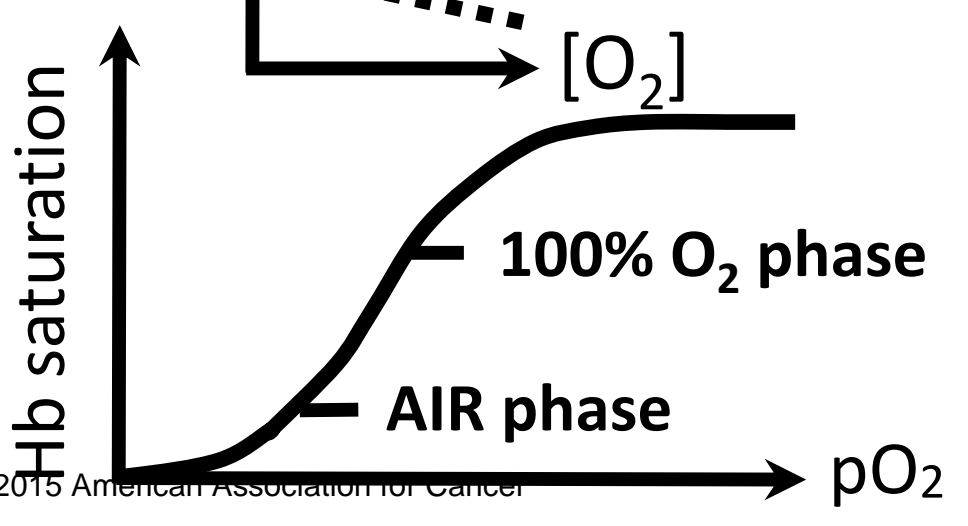




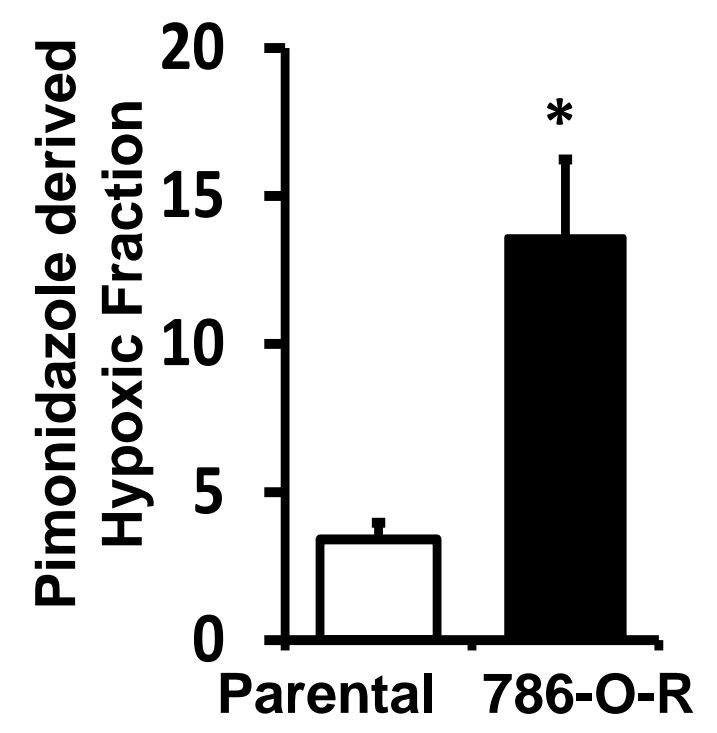

C

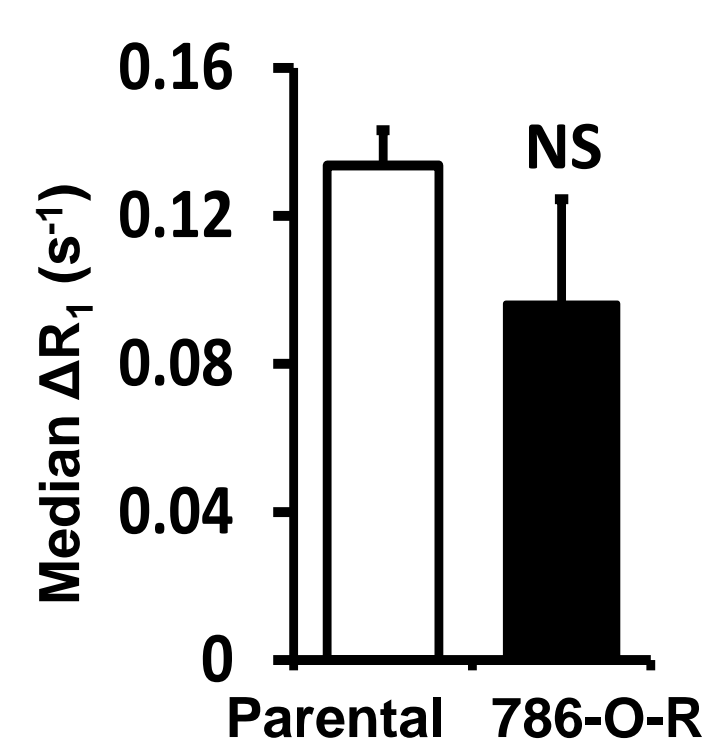

D

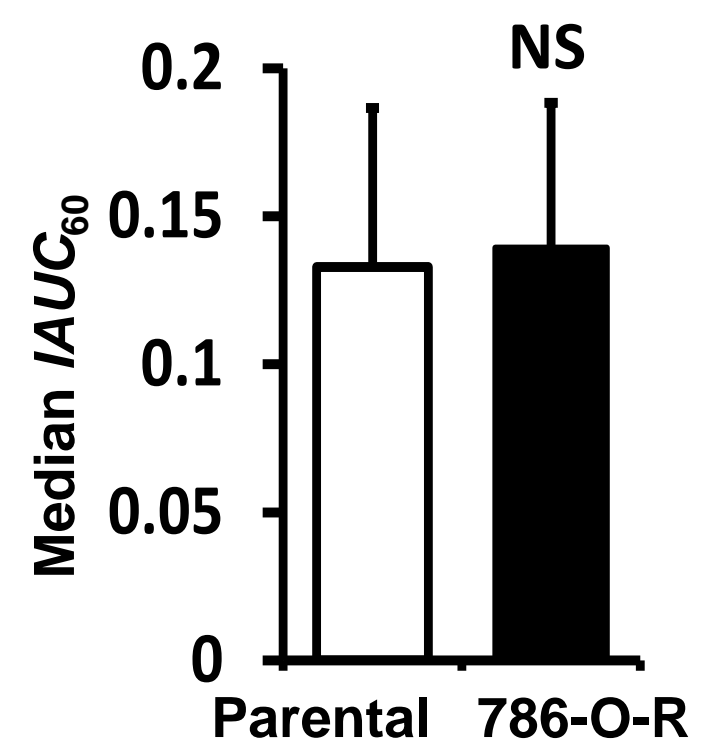

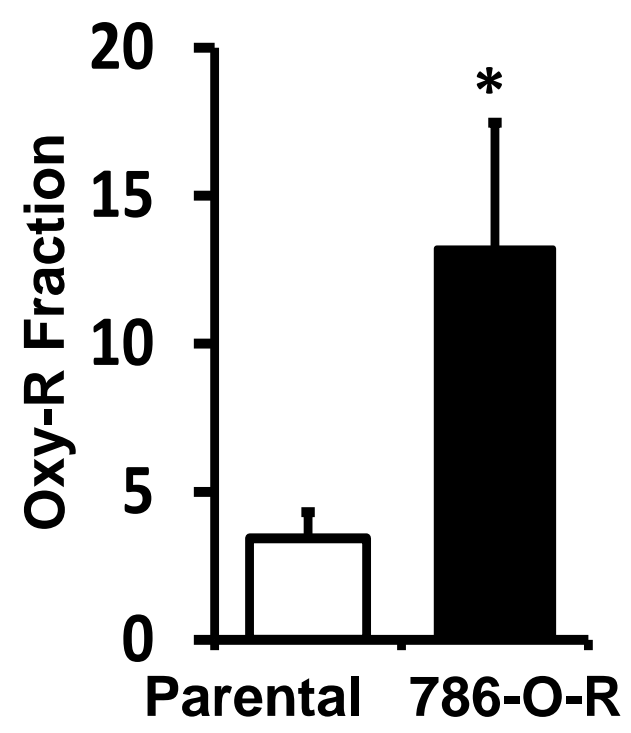

E

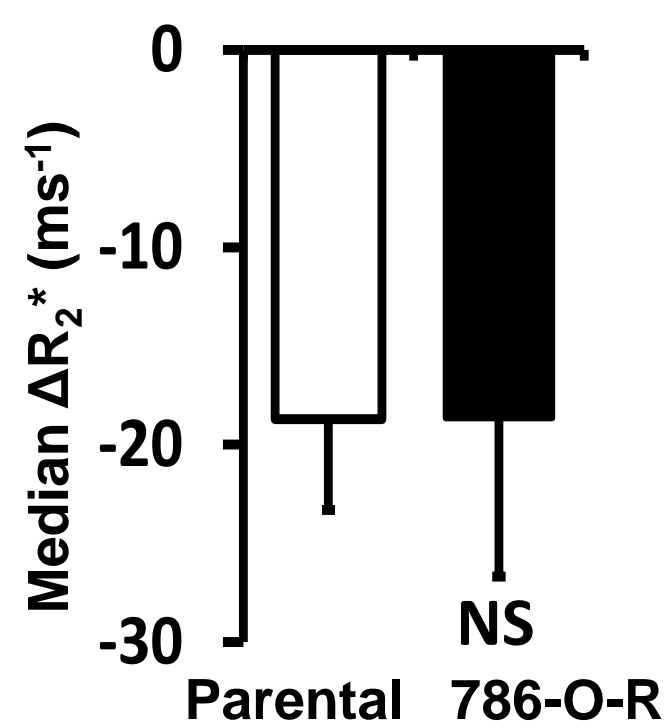


FIGURE 4

A $786-0-R$

\section{OE-MRI}

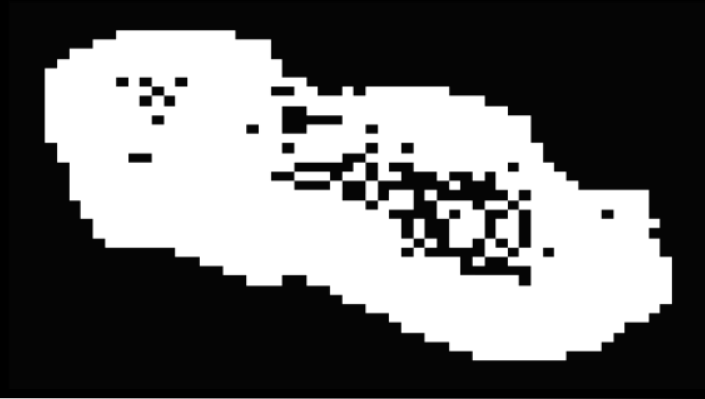

\section{Combined MRI}
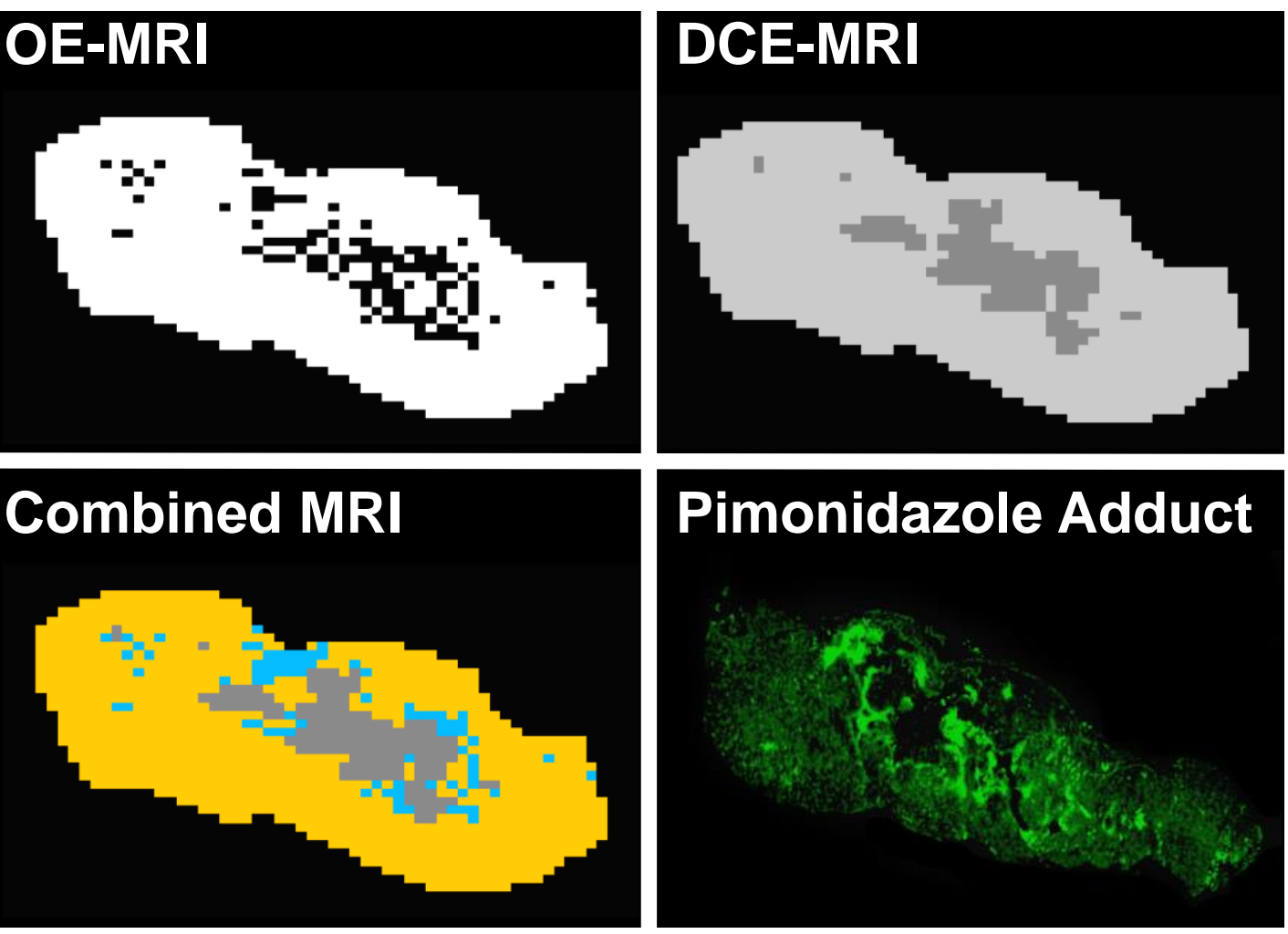

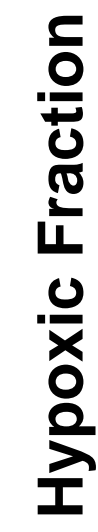

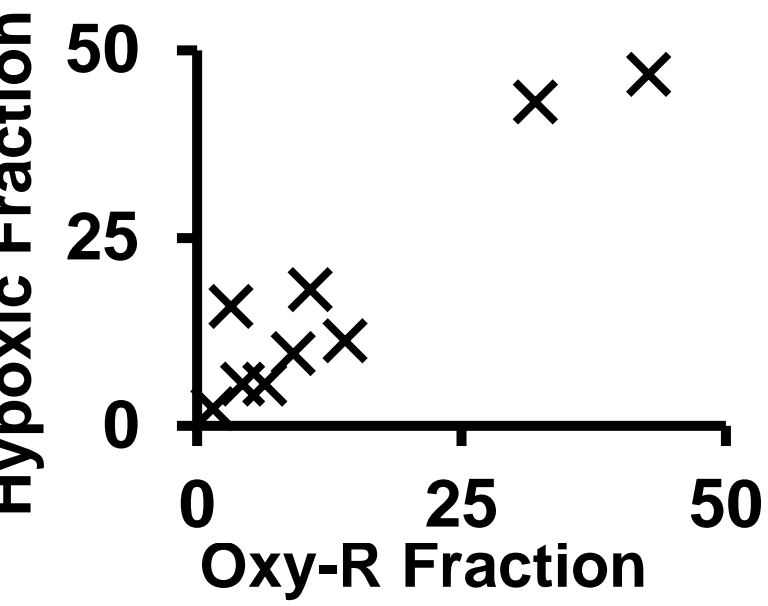

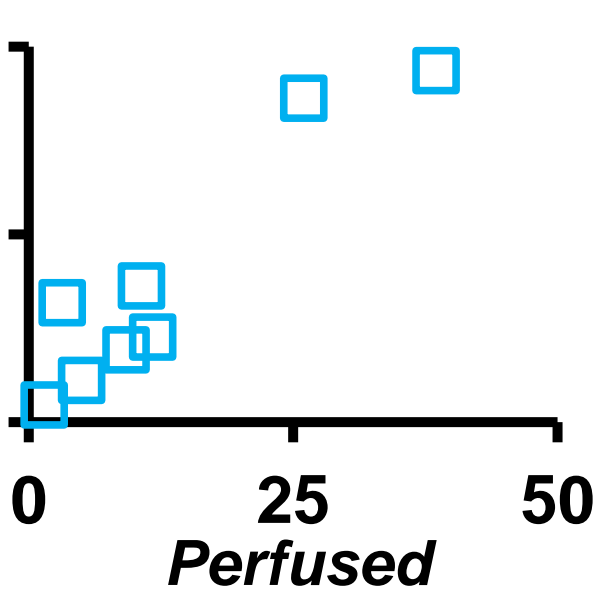

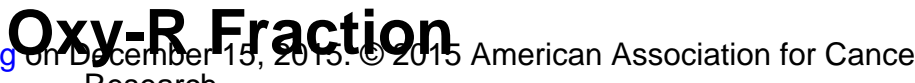

B $5 W 620$
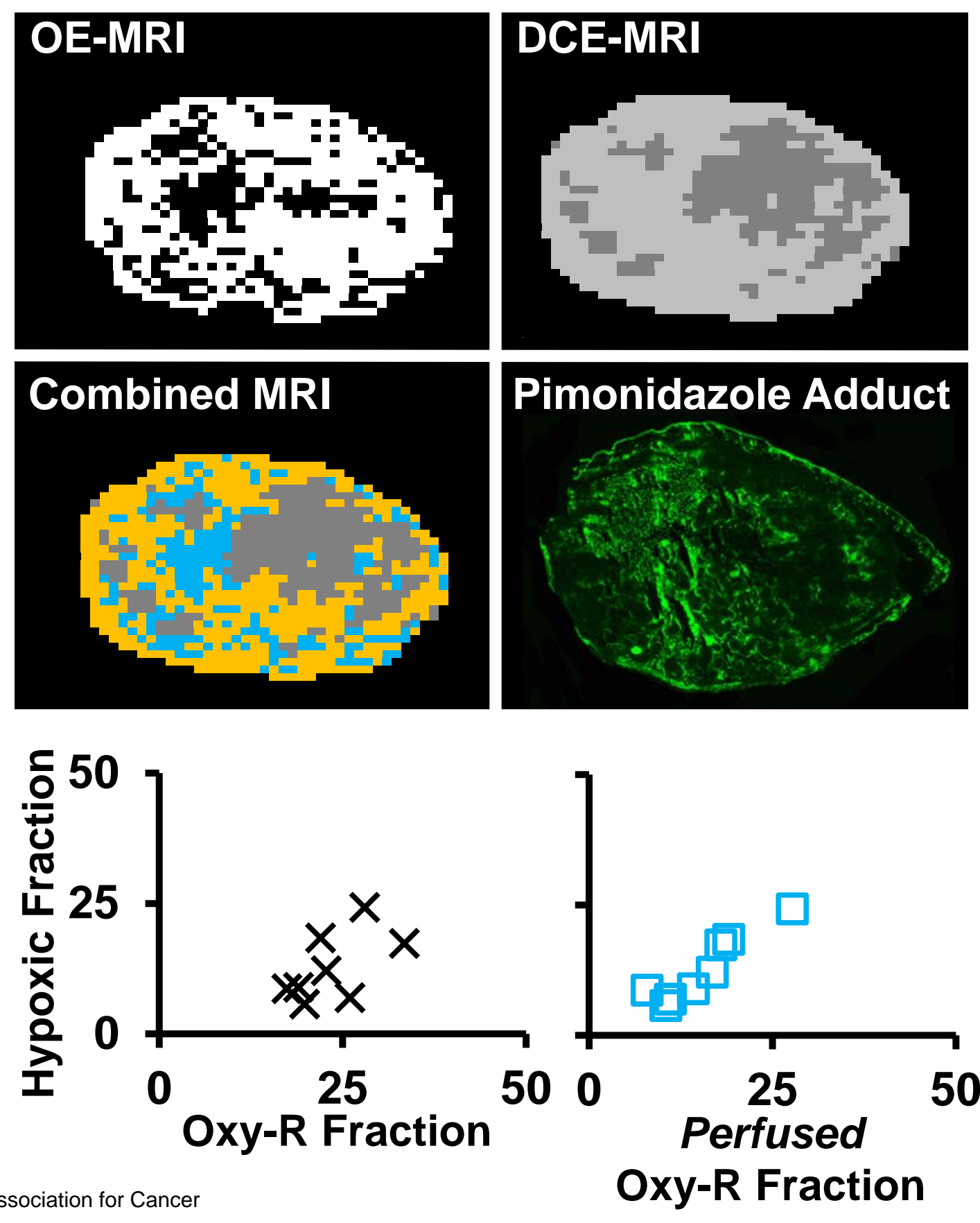
A $\quad$ Air $100 \% \mathrm{O}_{2} \quad$ Air $\quad 100 \% \mathrm{O}_{2} \quad$ B
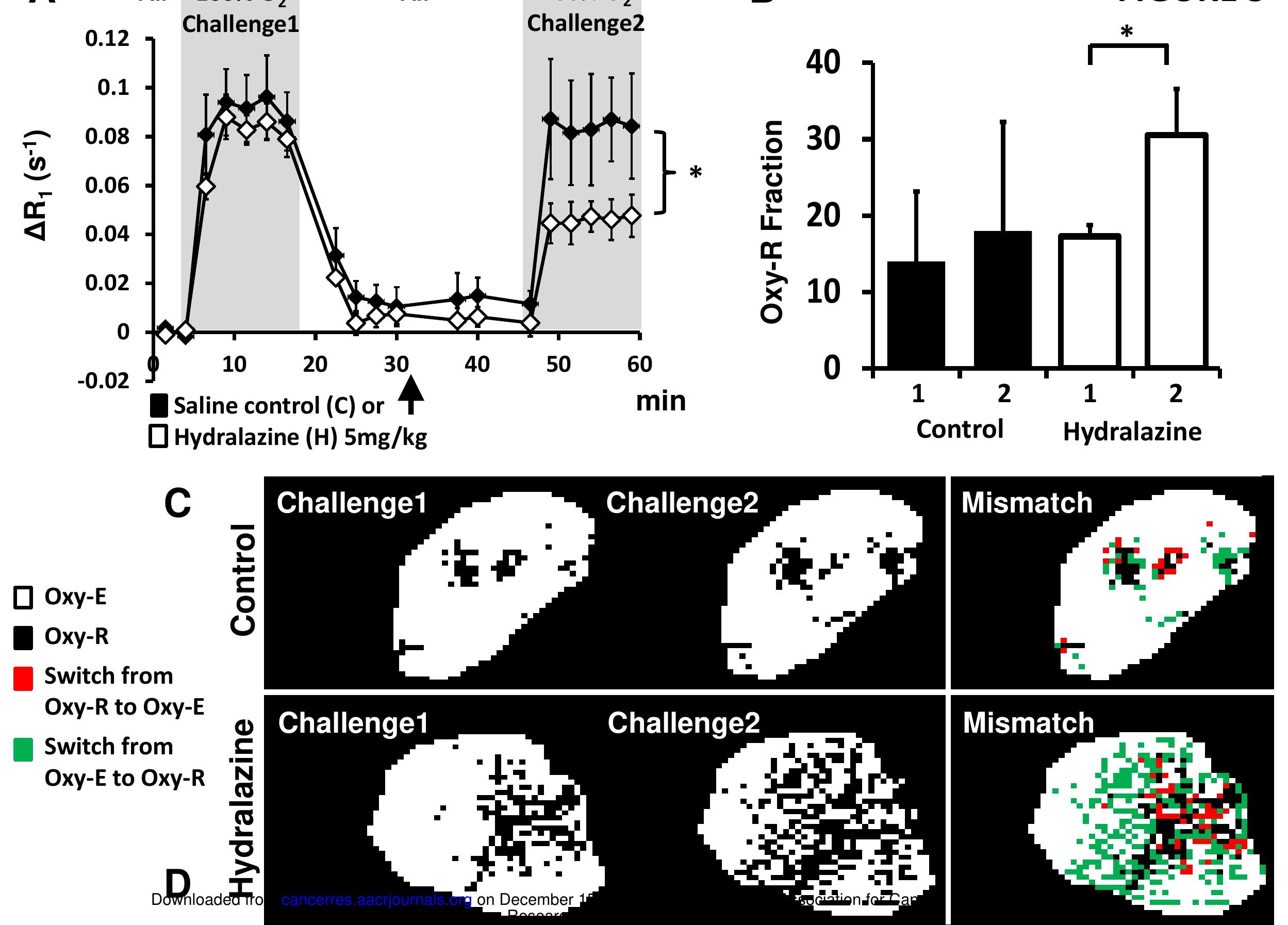


\section{Oxygen enhanced MRI accurately identifies, quantifies, and maps hypoxia in preclinical cancer models}

James P B O'Connor, Jessica KR Boult, Yann Jamin, et al.

Cancer Res Published OnlineFirst December 9, 2015.

Updated version Access the most recent version of this article at: doi:10.1158/0008-5472.CAN-15-2062

Supplementary Access the most recent supplemental material at:

Material http://cancerres.aacrjournals.org/content/suppl/2015/12/08/0008-5472.CAN-15-2062.DC1.html

Author Author manuscripts have been peer reviewed and accepted for publication but have not yet been Manuscript edited.

\section{E-mail alerts Sign up to receive free email-alerts related to this article or journal.}

Reprints and To order reprints of this article or to subscribe to the journal, contact the AACR Publications Subscriptions Department at pubs@aacr.org.

Permissions To request permission to re-use all or part of this article, contact the AACR Publications Department at permissions@aacr.org. 